\title{
Experimental Study of a Non-Thermal Food Processing System Using a Series-Connected Bipolar Voltage Multiplier with Multiple Electrodes
}

\author{
Kei Eguchi ${ }^{\mathrm{a},}{ }^{,}$, Kanji Abe ${ }^{\mathrm{a}}$, Ryo Ogata ${ }^{\mathrm{a}}$, Ichirou Oota ${ }^{\mathrm{b}}$ \\ ${ }^{a}$ Fukuoka Institute of Technology, 3-30-1 Wajirohigashi, Higashi-ku, Fukuoka, 811-0295 Japan \\ ${ }^{b}$ National Institute of Technology, Kumamoto College, 2659-2 Suya, Koushi-shi, Kumamoto, 861-1102 Japan \\ *Corresponding Author: eguti@fit.ac.jp
}

\begin{abstract}
To provide nutritious and fresh processed foods at low cost, non-thermal food processing utilizing an underwater shockwave is one of the most promising methods. In the non-thermal food processing system, a high voltage multiplier is the vital component to generate the underwater shockwave. In this paper, an experimental study is conducted concerning a non-thermal food processing system using a series-connected bipolar voltage multiplier with multiple electrodes. In the proposed system, a target food is destroyed from both sides by underwater shockwaves. Therefore, unlike conventional systems, the proposed system can soften the whole flesh of target foods effectively. Furthermore, owing to the series-connected bipolar topology, the proposed multiplier can achieve not only higher speed but also higher step-up gain than conventional multipliers. Therefore, the proposed system can process the target food at high speed. The feasibility of the proposed system is clarified by experiments.
\end{abstract}

Keywords: non-thermal food processing, high voltage multipliers, Cockcroft-Walton multipliers, underwater shockwaves, multiple electrodes.

\section{Introduction}

In the aging society such as Japan, nutritious and fresh processed foods are required for elderly persons. To provide nutritious and fresh processed foods at low cost, non-thermal food processing utilizing an underwater shockwave ${ }^{(1),(2)}$ is one of the most promising methods. By utilizing the underwater shockwave, this method destroys the cell wall and organization of foods without heating. The non-thermal food processing system is mainly composed of a high voltage multiplier, a pressure vessel, a high voltage relay, and a big capacitor. The operation principle of the non-thermal food processing system is as follows: First, a high stepped-up voltage is generated by a high voltage multiplier, where the high stepped-up voltage is charged to an external big capacitor. Next, the electric charge stored in the big capacitor is discharged from two electrodes by turning on the high voltage relay. Then, the stored energy in the external capacitor turns into shockwave energy in the pressure vessel. Finally, the target food is crushed by the underwater shockwave. Therefore, the key component of this system is the high voltage multiplier ${ }^{(3)-(10)}$ for generating underwater shockwaves.

In past studies, one of the most popular high voltage multipliers is the Cockcroft-Walton voltage multiplier ${ }^{(3),(4)}$ (CWVM). Unlike a high voltage generator using a transformer with high turn ratio, the traditional CWVM can offer light and small circuit configuration. For this reason, several types of high voltage multipliers have been proposed: Bipolar voltage multiplier ${ }^{(5)-(7)}$, High-speed bipolar multiplier ${ }^{(8),(9)}$, series-connected bipolar multiplier ${ }^{(10)}$, and so on. However, the conventional voltage multipliers suffer from low speed and low gain. Therefore, the speed of the non-thermal food processing system using conventional voltage multipliers is slow. Furthermore, it is difficult to process the whole flesh of the target food such as fruits, meats, etc., because the softened part of the target food depends on the position of the electrodes.

In this paper, in order to process target foods such as fruits, meats, etc., an experimental study is conducted concerning the non-thermal food processing system using a series-connected bipolar voltage multiplier with multiple electrodes. In the proposed system, the target food is destroyed effectively from both sides by the electric 
discharge from four electrodes. For this reason, unlike conventional systems, the proposed system can soften the target food effectively. Furthermore, owing to the series-connected bipolar topology, the proposed multiplier can achieve not only higher speed but also higher step-up gain than conventional multipliers. Therefore, the proposed system can process the target food at high speed. To clarify the feasibility of the proposed system, experimental evaluation is performed concerning the laboratory prototype.

The rest of this paper is organized as follows. In section 2 , the problem definition of this research is described. In section 3, the circuit configuration and operation principle of the proposed system are explained. To confirm the validity of the proposed system, experimental results are shown in Section 4. Finally, conclusion and future work are drawn in section 5 .

\section{Problem Definition}

Figure 1 illustrates the architecture of the conventional non-thermal food processing system utilizing an underwater shockwave ${ }^{(10)}$. In the non-thermal food processing system of figure 1, first, a high stepped-up voltage is generated by a high voltage multiplier, where the high stepped-up voltage is charged to an external big capacitor. Next, the electric charge stored in the external big capacitor is discharged from two electrodes by turning on a high voltage switch. Then, the stored energy in the external capacitor turns into shockwave energy in the pressure vessel. Finally, the target food is crushed by spalling destruction. In this food processing system, the water-rich food can be processed easily by small energy.

Figure 2 shows an example of the pressure vessel to fill water, where a pressure cooker is employed to process an apple. As figures 1 and 2 show, two electrodes were located near the target food. However, it is difficult to process the whole flesh of the target food such as fruits, meats, etc. The softened part of the target food depends on the position of the electrodes as shown in figure 3 . To process the entire flesh of the target food such as fruits, the non-thermal food processing system using a series-connected bipolar voltage multiplier with multiple electrodes is proposed in this paper. Figure 4 illustrates the architecture of the proposed non-thermal food processing system. Unlike the conventional systems of figure 1 , the high voltage multiplier of the proposed system has multiple electrodes. Therefore, the target food is destroyed from both sides by the electric discharge from four electrodes. For this reason, unlike conventional systems, the proposed system can process the target food effectively. Furthermore, owing to the series-connected bipolar topology, the proposed multiplier can achieve not only higher speed but also higher step-up gain than conventional multipliers. Therefore, the proposed system can process the target food at high speed.

In the following section, the features of the high voltage multiplier used in the proposed system will be discussed.

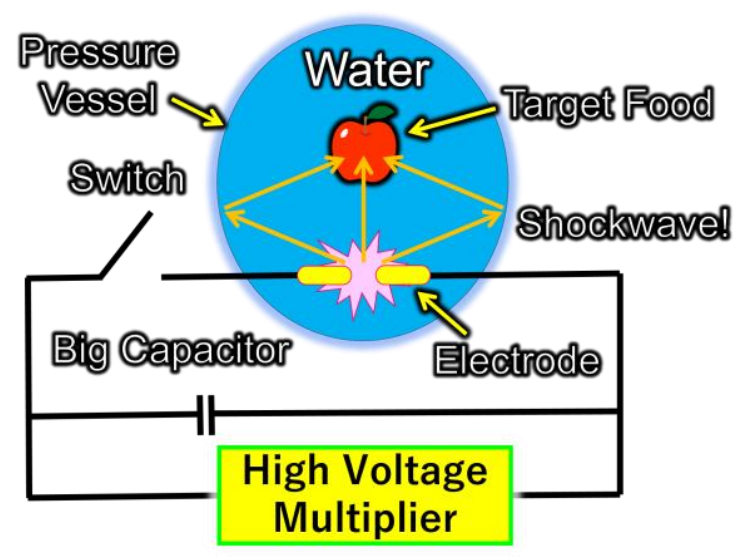

Fig. 1. Architecture of the previous non-thermal food processing system utilizing an underwater shockwave ${ }^{(10)}$.

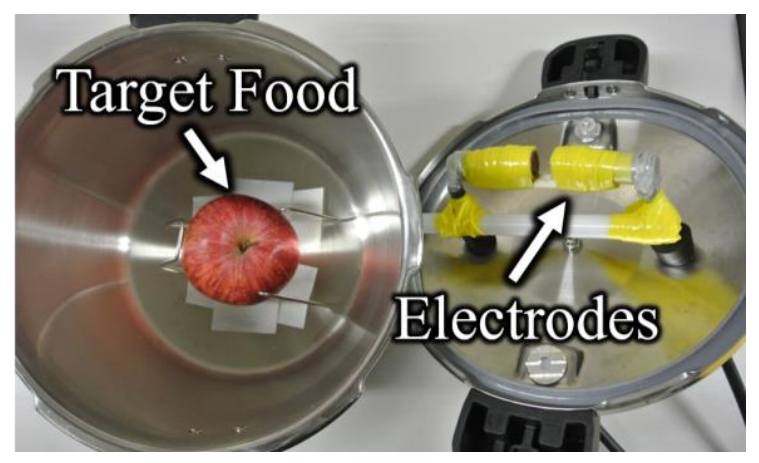

Fig. 2. An example of the pressure vessel.

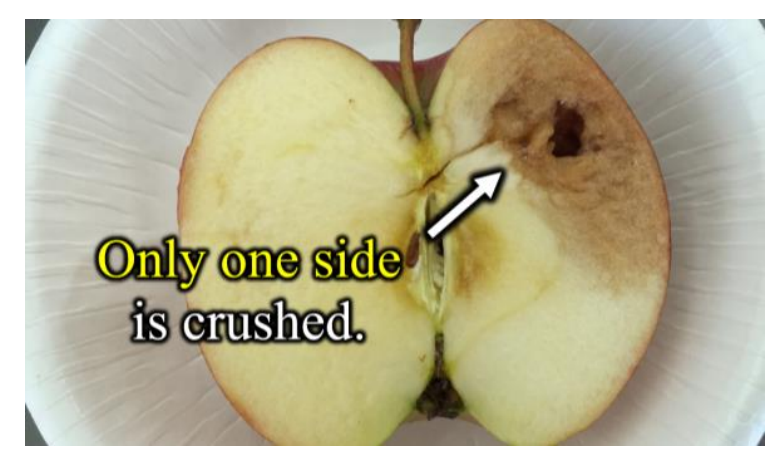

Fig. 3. Apple processed by the conventional system. 


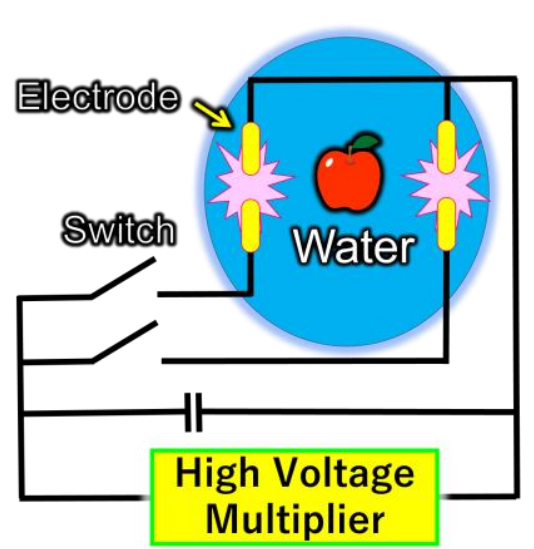

Fig. 4. Architecture of the proposed non-thermal food processing system utilizing an underwater shockwave.

\section{High Voltage Multiplier}

\subsection{Block Diagram}

Figure 5 depicts the comparison of the block diagrams between the proposed multiplier and conventional multipliers. As figure 5 shows, four types of high voltage multipliers are compared with the proposed multiplier.

Figure 5 (a) illustrates the traditional Cockcroft-Walton multiplier. As figure 5 (a) shows, the traditional high voltage multiplier ${ }^{(3),(4)}$ consists of a positive voltage multiplier block. The benefit of the traditional multiplier is the simple circuit configuration. However, due to the threshold voltage drop caused by diode switches, the step-up gain becomes small.

Following this study, the bipolar voltage multiplier ${ }^{(5)-(7)}$ of figure 5 (b) was proposed. The bipolar voltage multiplier is composed of a positive voltage multiplier block and a negative voltage multiplier block. By combining the outputs of these blocks, a high voltage across an output load is offered as an output. Therefore, owing to the bipolar topology, the threshold voltage drop can be alleviated by the bipolar voltage multiplier. However, due to the switching control using ac inputs, the response speed of the bipolar voltage multiplier is slow.

To overcome this problem, the high-speed bipolar voltage multiplier ${ }^{(8),(9)}$ of figure 5 (c) was developed. The high-speed multiplier consists of a positive and a negative voltage multiplier blocks and a full waveform rectifier. By driving these voltage multiplier blocks by high-speed rectangular clock pulses, a high voltage across the output load is provided. Owing to the switching control using high speed rectangular pulses, the high-speed multiplier can

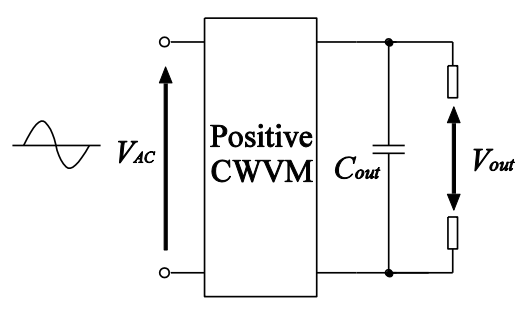

(a) Cockcroft-Walton multiplier ${ }^{(3),(4)}$

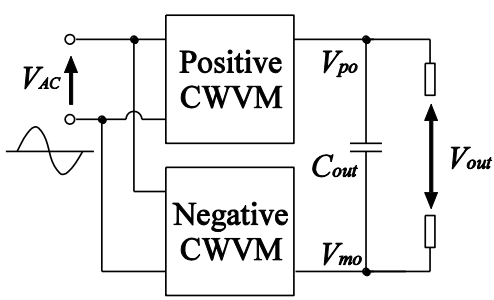

(b) Bipolar voltage multiplier ${ }^{(5)-(7)}$.

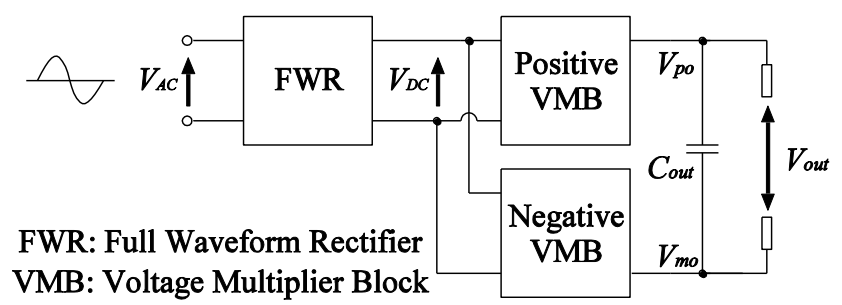

(c) High-Speed bipolar voltage multiplier ${ }^{(8),(9)}$.

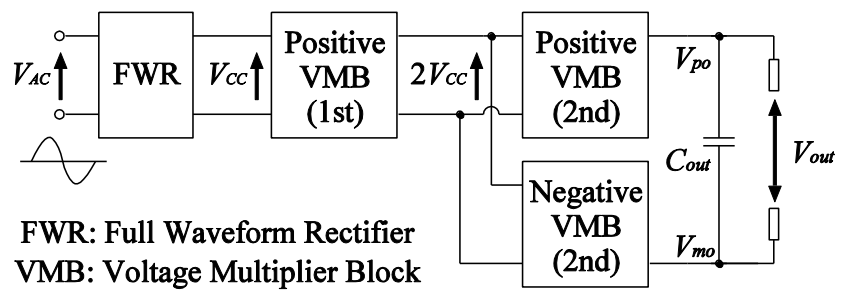

(d) Series-connected bipolar voltage multiplier ${ }^{(10)}$.

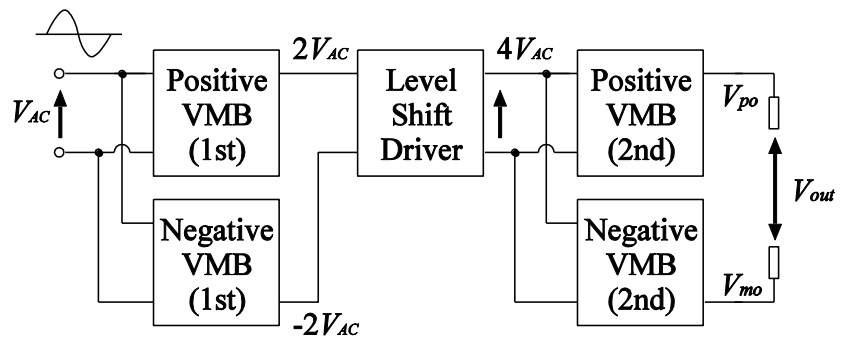

(e) Proposed multiplier.

Fig. 5. Comparison of high voltage multipliers.

achieve high response speed. However, the step-up gain of the high-speed multiplier was not improved from conventional converters.

To achieve high step-up gain, the series-connected high voltage multiplier ${ }^{(10)}$ of figure 5 (d) was suggested. The series-connected multiplier consists of a full waveform 
rectifier, two positive voltage multiplier blocks, and a negative voltage multiplier block. By connecting these voltage multiplier blocks in series, the input voltage is amplified twice in this multiplier. Owing to the series-connected topology, the series-connected multiplier can achieve high step-up gain. However, there is still room for improvement in step-up gain.

Figure 5 (e) illustrates the block diagram of the proposed multiplier. The proposed multiplier consists of two positive multiplier blocks, two negative multiplier blocks, and a level shift driver. First, the ac input is amplified by the 1st positive and negative multiplier blocks. Then, the level shift driver generates high-speed rectangular pulses by combining the outputs of these 1st multiplier blocks, where the amplitude of the rectangular pulses becomes $4 V_{A C}$. Finally, by amplifying the amplitude of the high-speed rectangular pulses by the 2nd multiplier blocks, the bipolar output voltages are offered to output loads. Therefore, the proposed multiplier can achieve not only high speed but also high step-up gain.

\subsection{Circuit Configuration}

Figure 6 depicts the circuit configuration of the proposed high voltage multiplier. As figure 6 shows, the proposed multiplier is controlled by non-overlapped two-phase clock pulses $\Phi_{1}$ and $\Phi_{2}$. By combining the output voltages $V_{p o}$ and $V_{m o}$, the proposed multiplier offers the following high stepped-up voltage:

$$
V_{\text {out }}=28 V_{\text {max }}-36 V_{\text {th }} \text {, }
$$

where $V_{\max }$ denotes the maximum value of $V_{A C}$ and $V_{t h}$ denotes the threshold voltage of the diode switch. In the proposed multiplier, the step-up gain is set to 28 in order to

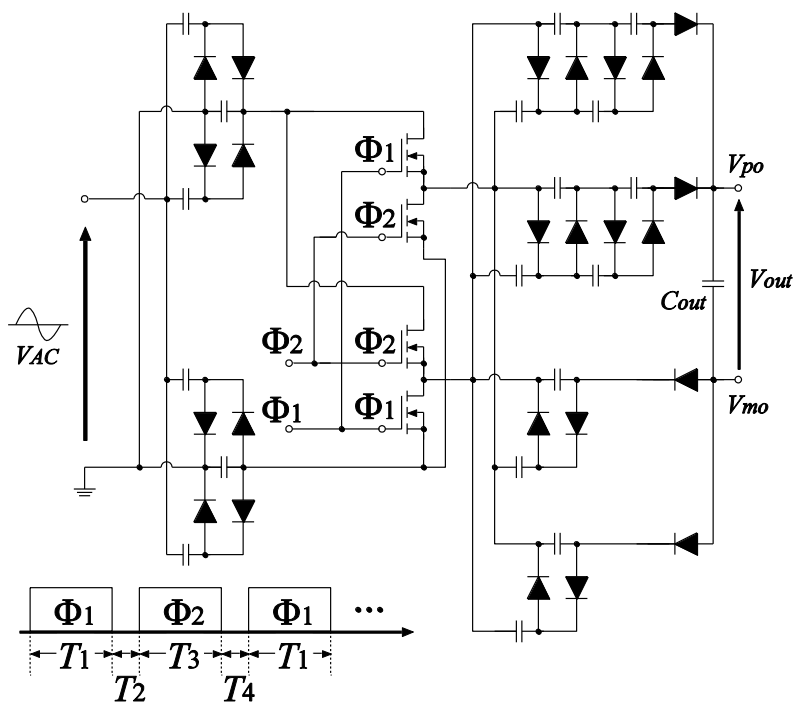

Fig. 6. Proposed high voltage multiplier.
Table 1. Comparison of output voltages.

\begin{tabular}{|l|c|}
\hline & Output Voltage \\
\hline Traditional CWVM & $V_{\text {out }}=2 N\left(V_{\max }-V_{\text {th }}\right)$ \\
\hline $\begin{array}{l}\text { Bipolar high voltage } \\
\text { multiplier }\end{array}$ & $V_{\text {out }}=2 N\left(V_{\max }-V_{\text {th }}\right)+2 N V_{\max }-(2 N+1) V_{\text {th }}$ \\
\hline $\begin{array}{l}\text { Series-connected high } \\
\text { voltage multiplier }\end{array}$ & $\begin{array}{r}V_{\text {out }}=2(2 N+1)\left(V_{\max }-V_{\text {th }}\right) \\
+2(2 N)\left(V_{\max }-V_{\text {th }}\right)-V_{\text {th }}\end{array}$ \\
\hline Proposed multiplier & $\begin{array}{r}V_{\text {out }}=2(4 N+1)\left(V_{\max }-V_{\text {th }}\right)-(2 N+1) V_{\text {th }} \\
+2(2 N+1)\left(V_{\max }-V_{\text {th }}\right)-(N+1) V_{\text {th }}\end{array}$ \\
\hline
\end{tabular}

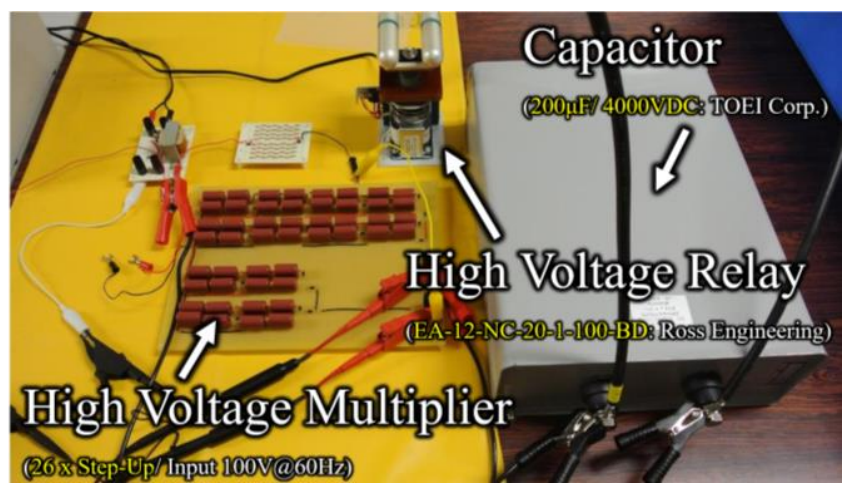

Fig. 7. Laboratory prototype of the proposed system.

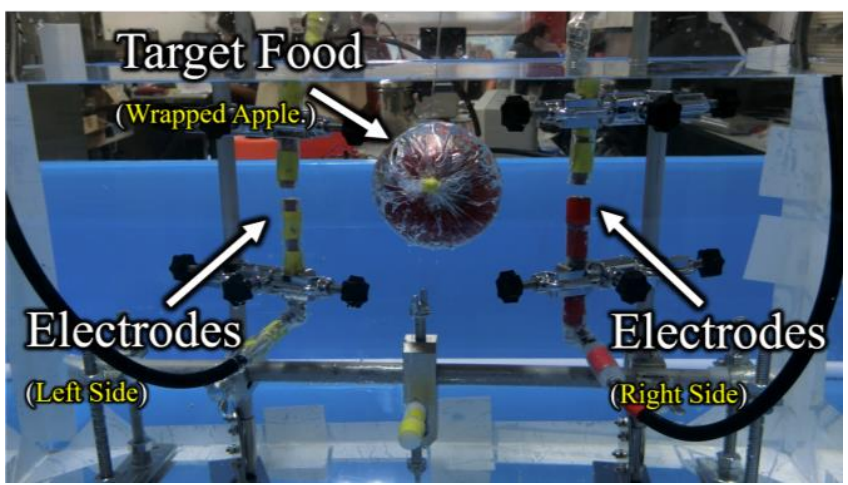

Fig. 8. Acrylic water tank to investigate discharging process.

generate about 4kV from 100V@60Hz, because it is known that a $3.5 \mathrm{kV}$ output is necessary to destroy fruits such as apples, tomato, etc.

Table 1 demonstrates the comparison of output voltages between the proposed multiplier and conventional multipliers. In Table $1, N(=1,2, \ldots)$ is the number of stages of the voltage multiplier block. As Table 1 shows, the proposed multiplier can achieve higher gain than others.

\section{Experiment}




\subsection{Experimental Setup}

To clarify the feasibility of the proposed system, experiments were conducted concerning the laboratory prototype shown in figure 7 . The laboratory prototype of figure 7 consists of a high voltage multiplier, a high voltage relay, and a big capacitor. In figure 7 , the high voltage multiplier was built with commercially available components on PCB boards, where the step-up gain is 28 and the input voltage is $100 \mathrm{~V} @ 60 \mathrm{~Hz}$. The stepped-up voltage generated from the high voltage multiplier is provided to the output capacitor. The size of the output capacitor produced by TOEI corporation is $200 \mu \mathrm{F}$ and the rated voltage is $4000 \mathrm{~V} \mathrm{DC}$, where the capacitor is made by using polypropylene film and insulating oil. The connection between the high voltage multiplier and the output capacitor is controlled by the high voltage relay produced by Ross Engineering, where the HV contact of the high voltage relay is $12 \mathrm{kV}$ and insulation to ground is $20 \mathrm{kV}$. Figure 8 shows the chamber to fill water. In experiments, the acrylic water tank was used to investigate discharging process of the non-thermal food processing. The size of the acrylic water tank is $60 \times 30 \times 36 \mathrm{~cm}$. As figure 8 shows, four electrodes were set in water. Between these electrodes, the wrapped apple is fixed as shown in figure 8 , because the effect of underwater shockwaves can be confirmed by the color change of the apple flesh.

\subsection{Experimental Results}

Figure 9 demonstrates the charging process of the $200 \mu \mathrm{F}$ capacitor. In figure 9 , the red line shows the output voltage of the positive converter block and the green line shows the output voltage of the negative converter block. As you can see from figure 9, the settling time is $281 \mathrm{sec}$. and the output voltage is about $3.98 \mathrm{kV}$. On the other hand, the settling time of the traditional CWVM was more than $20 \mathrm{~min}$. for charging the $200 \mu \mathrm{F}$ capacitor.

Figure 10 demonstrates the discharging process captured by the high speed camera EX-100PRO. In the experiment of figure 10, the gap between copper electrodes was set to $10 \mathrm{~mm}$ and a wrapped target food was located near the electrodes. As figure 10 shows, first, the water around the copper electrodes evaporates by electric discharge. Then, shockwaves occur by implosion. As you can see from figure 10 , the color of flash light is green, because the copper electrodes are melted by electric discharge. Therefore, the water in the acrylic water tank is contaminated by copper. In a future study, we are going to investigate the copper contamination of water.

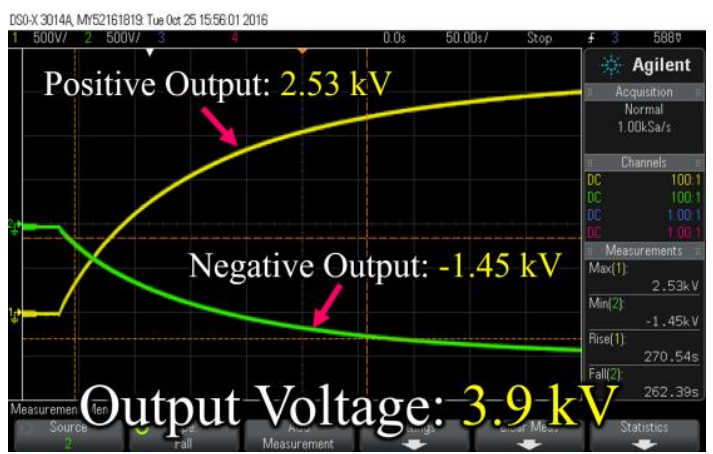

(a) Measured outputs of the positive/negative converter blocks.

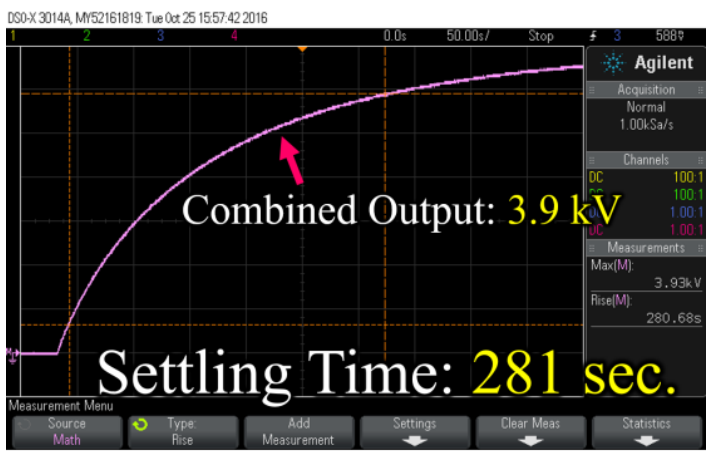

(b) Combined output voltage.

Fig. 9. Measured charging process of a $200 \mu \mathrm{F}$ capacitor.

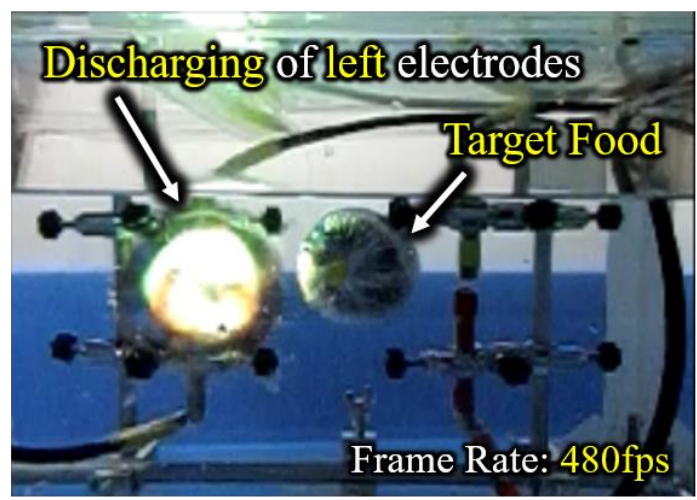

(a) Left electrodes.

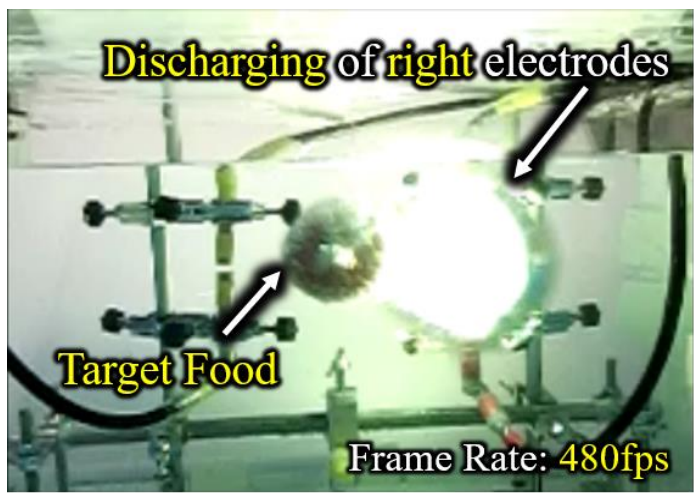

(b) Right electrodes.

Fig. 10. Measured discharging process. 


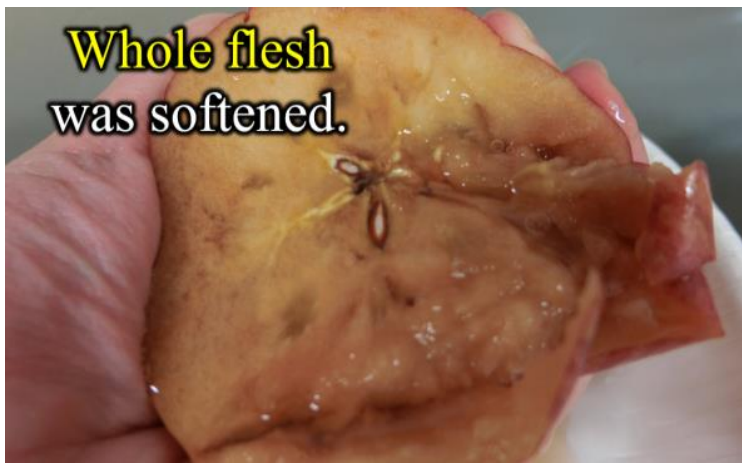

Fig. 11. Cross section of the processed food.

Figure 11 shows the cross section of the processed food by the proposed system. As figure 11 shows, the whole flesh of the target food is softened by underwater shockwaves. Concretely, the hardness of the target food was changed from $53.8 \mathrm{~N}$ to $30.4 \mathrm{~N}$ by the non-thermal food processing. Of course, the proposed non-thermal food processing system can completely crush the target food by increasing the number of discharging processes.

\section{Conclusions}

A non-thermal food processing system using a series-connected bipolar voltage multiplier with multiple electrodes has been proposed in this paper. The feasibility of the proposed system was confirmed by the experiments using a laboratory prototype.

The results of this study are as follows: 1 . The settling time of the proposed system was less than $281 \mathrm{sec}$. for charging a $200 \mu \mathrm{F}$ capacitor. 2 . The whole flesh of the target food was softened by underwater shockwaves. By the proposed non-thermal food processing, the hardness of the processed food was changed from $53.8 \mathrm{~N}$ to $30.4 \mathrm{~N}$. From this result, the proposed system can offer nutritious and fresh processed foods. 3. Due to high voltage discharging processes, the copper electrodes were melted. Therefore, the copper contamination of water must be investigated in a future study. This study is still going on. Now, we are going to develop health functional foods by injecting vitamins, alcohols, and so on. The proposed system will open up new vista in the field of the food processing.

\section{Acknowledgement}

This research is supported by a grant from Urakami Foundation for Food and Food Culture Promotion.

\section{References}

(1) Kazuyuki Naha, Ken Shimojima, Yoshitaka Miyafuji, Shigeru Itoh: "Design and development of pressure vessel for improvement of manufacturing rice-powder efficiency using underwater shock wave", Proc. of the ASME 2012 Pressure Vessels and Piping Conf., pp. 15-19, 2012.

(2) Shuhei Shinzato, Yoshikazu Higa, Tatsuhiro Tamaki, Hirofumi Iyama, Shigeru Itoh: "Computational simulation of underwater shock wave propagation using smoothed particle hydrodynamics", Materials Science Forum, vol. 767, pp. 86-91, 2013.

(3) A. Lamantia, P. Maranesi, L. Radrizzani, "The dynamics of the Cockcroft-Walton voltage multiplier", Proc. of the IEEE Power Electronics Specialists Conf., pp. 485-490, 1990.

(4) Jianing Wang, Sjoerd W.H. de Haan, J.A. Ferreira, Peter Luerkens: "Complete model of parasitic capacitances in a cascade voltage multiplier in the high voltage generator", Proc. of the IEEE ECCE Asia Downunder, pp. 18-24, June 2013.

(5) Shahid Iqbal, Rosli Besar: "A bipolar CockcroftWalton voltage multiplier for gas lasers", American Journal of Applied Sciences, vol. 4, no.10, pp. 795-801, 2007.

(6) Kei Eguchi, Ichirou Oota, Shinya Terada, Hongbing Zhu: "2x/3x step-up switched-capacitor (SC) AC-DC converters for RFID tags", International Journal of Intelligent Engineering and Systems, vol. 4, no.1, pp. 1-9, 2011.

(7) Shahid Iqbal: "A hybrid symmetrical voltage multiplier", IEEE Trans. Power Electronics, vol. 29, no.1, pp. 6-12, 2014.

(8) Kei Eguchi, Sawai Pongswatd, Shinya Terada, Ichirou Oota: "Parallel-connected high voltage multiplier with symmetrical structure", Applied Mechanics and Materials, vol. 619, pp. 173-177, 2014.

(9) Kei Eguchi, Ichirou Oota, Shinya Terada, Kuniaki Fujimoto: "Design of an inductor-less bipolar voltage multiplier for high-voltage low-current applications", ICIC Express Letters Part B: Applications, vol.6, no.1, pp. 1-6, 2015.

(10) Kei Eguchi, Kanji Abe, Hiroki Fujisawa, Ichirou Oota: "Design of a high voltage multiplier with series-connected bipolar topology", Proc. of the Third Intl. Conf. on Advances in Applied Science and Environmental Technology, pp. 113-117, 2015. 